\title{
Germanica
}

\section{La régression comme double stratégie du détour et du retour dans Kali, eine Vorwintergeschichte de Peter Handke}

Die Regression als Strategie des Umwegs und der Rückkehr im Roman Kali: eine Vorwintergeschichte von Peter Handke

Regression as progression in Handke's novel Kali: eine Vorwintergeschichte

\section{Gauthier Labarthe}

\section{OpenEdition}

\section{Journals}

Édition électronique

URL : http://journals.openedition.org/germanica/4015

DOI : 10.4000/germanica.4015

ISSN : 2107-0784

\section{Éditeur}

Université de Lille

\section{Édition imprimée}

Date de publication : 31 décembre 2017

Pagination : 45-59

ISBN : 9782913857407

ISSN : 0984-2632

\section{Référence électronique}

Gauthier Labarthe, «La régression comme double stratégie du détour et du retour dans Kali, eine

Vorwintergeschichte de Peter Handke », Germanica [En ligne], 61 | 2017, mis en ligne le 01 janvier 2021, consulté le 02 mars 2021. URL : http://journals.openedition.org/germanica/4015 ; DOI : https:// doi.org/10.4000/germanica.4015 


\title{
La régression comme double stratégie du détour et du retour dans Kali, eine Vorwintergeschichte de Peter Handke
}

\author{
Gauthier LABARTHE \\ Université Toulouse Jean Jaurès
}

\begin{abstract}
Épopée, mystères, conte : ces catégories issues de la littérature médiévale sont de plus en plus utilisées par la critique handkéenne pour analyser les textes de ce que l'on pourrait qualifier de deuxième phase d'écriture chez Peter Handke. Malgré l'absence de rupture nette et précise dans sa production, force est de constater un tournant que l'on pourrait interpréter, à la suite de Heinke Wagner, comme un mouvement de conversion de la «théologie négative vers une ouverture aux mystères $»^{1}$ auxquels l'individu ne peut apporter de réponses. Face à l'énigme qui creuse le texte et menace la confiance restaurée dans le langage, l'auteur autrichien recourt au conte comme stratégie du détour, comme une tentative de réponse à l'innommable que constitue le traumatisme à la fois individuel et collectif. Cette perspective rejoint l'une des tendances actuelles de la psychanalyse à envisager le conte comme «stratégie du détour » pour expliquer le fonctionnement de ces récits dans la psychanalyse, en insistant sur leur valeur métaphorique qui permettrait à l'auditeur, par condensation métaphorisante, d'identifier
\end{abstract}

1. - Heinke Wagner, La Mission théâtrale de Peter Handke, Bern, Peter Lang, 2003, p. 5.

GERMANICA, 2017, LXI, pp. 45 à 59. 
ses propres affects, même les plus terribles, ainsi que des aspects de son psychisme tout en les mettant à une bonne distance. Si ces aspects doivent être pris en compte, ils ne suffisent cependant pas à la compréhension du sujet dans le roman Kali : une histoire d'avant-hiver.

Dans ce roman publié en 2007, Peter Handke raconte le voyage d'une chanteuse dans la cité saline de Kali. Vivant au milieu des réfugiés ayant fui la Troisième Guerre mondiale qui fait rage, elle va alors se mettre à la recherche d'un enfant perdu. Il apparaît très rapidement que le roman présente de nombreuses caractéristiques du conte. Parmi elles, on retrouve la figure du conteur, le symbolisme du chemin, l'atemporalité et l'indétermination spatiale produite par le merveilleux, l'onirisme et les métamorphoses. Il s'agira alors de voir dans quelle mesure ces moyens sont mis au service d'un mouvement de régression, de retour symbolique vers soi et les contrées archaïques de l'enfance, ce point originaire enseveli par le deuil de la catastrophe individuelle (l'enfant perdu) et collective (la guerre mondiale). Face au poids de la responsabilité et au sentiment de la faute, Peter Handke recourt au conte avant tout comme méthode pour ouvrir le texte à une connaissance et une exploration de l'être. Le détour ici prend la forme d'un retour si l'on considère le conte comme une forme épique traditionnelle et pré-moderne, une forme élémentaire de la littérature qu'il faut envisager non pas comme la catégorie générique bien définie et codifiée que l'on connaît, mais comme une pratique, un mode de pensée primitif proche des formes simples et archétypiques de la psyché humaine.

Pour ce faire, il faudra retracer cette quête de l'archê ainsi que les différentes opérations dont elle procède. Interrogeant tout d'abord l'instance narratrice, nous verrons que l'auteur travaille à son propre effacement. Il s'emploie à disparaître hors de son dit pour instituer à travers un « je »-narrateur la figure d'une voix impersonnelle, celle du conteur, indiscernablement sienne et autre, refluant vers son archaïque commencement. Nous verrons que ce n'est qu'au prix de cette opération d'effacement que le passé, l'absent et l'objet perdu peuvent alors faire retour dans le monde présent des vivants. Exhumé des replis insondables de l'être et métamorphosé par le travail du rêve et de la figurabilité, ce qui avait été exclu de la sphère de l'énonciation est à nouveau présent dans l'imaginaire de la fiction et l'indétermination merveilleuse du conte.

\section{Un monde endeuillé}

Le conte se présente tout d'abord pour Peter Handke comme une manière de lutter contre l'oubli et de réparer ce qui a été perdu. Comme souvent dans ces textes, l'histoire prend sa source dans le surgissement d'une angoisse primaire et d'une expérience traumatique. La démarche d'écriture handkéenne s'apparente en effet à un travail de deuil qui s'ori- 
gine dans un sentiment de culpabilité né d'une mémoire trop lourde à porter et que l'auteur exprime dès les premières lignes: "Ầ moi aussi elle m'a fait peur, me fait peur. Mais je voudrais lui faire face. Peu à peu la mémoire s'enclenche et je l'entends, sans encore la voir $»^{2}$. Lorsque le narrateur évoque ici sa propre expérience de la mémoire, il désigne une expérience du surgissement qui est dessaisissement du sujet face à ce qui le dépasse. Cette mémoire apparaît en effet sur le mode singulier du choc ou de l'intrusion, à la manière de ces manifestations d'arrivance évoquées par Derrida et qui, conformément à la loi de l'hétéronomie ${ }^{3}$, provoque et accentue chez la chanteuse, pourtant de retour sur les terres de son enfance, le sentiment d'avoir perdu son identité et de ne pas appartenir à cette communauté au point de n'être plus capable d'identifier les siens : «J'étais chez les miens, et je n'ai pas reconnu les miens, moi ! Et qu'est-ce qui est inaltérable par exemple ? Les cuvettes pour moineaux dans le sable $»^{4}$. La femme exprime ici quelque chose qui la dépasse, quelque chose qui vient d'ailleurs, d'un Autre qui impose son obscure volonté. L'angoisse qui s'insinue, qui envahit de son malaise vague le personnage, renvoie à l'inquiétante étrangeté, c'est-à-dire à cet intime qui surgit comme étranger, inconnu, autre absolu, au point d'en être effrayant : c'est le familier étrange, le non familier intime.

Cette expérience vient de ce que les images issues des souvenirs, de ce passé qui ne lui appartient pas ou plus, ne sont pas accessibles sous une forme cohérente, compréhensible ni même représentable. Bien au contraire, issues de la subjectivité de la chanteuse, elles sont elles aussi excédentaires et opacifient en cela leur contenu. Elles relèvent d'une vision d'angoisse travaillée par l'altération et l'informe :

Il y a là une horrible découverte, celle de la chair qu'on ne voit jamais, le fond des choses, l'envers de la face, du visage, les sécrétats par excellence, la chair dont tout sort, au plus profond même du mystère, la chair en tant qu'elle est souffrante, qu'elle est informe, que sa forme par soi-même est quelque chose qui provoque l'angoisse. Vision d'angoisse, dernière révélation du tu es ceci - Tu es ceci qui est le plus loin de toi, ceci qui est le plus informe ${ }^{5}$.

Prisonnière de sa dialectique et de l'économie psychique du deuil et du désir, l'image vacille entre son cône d'ombre et sa vérité excédentaire.

2. - Peter Handke, Kali : une histoire d'avant-hiver, trad. Georges-Arthur Goldschmidt, Paris, Gallimard, 2011.

3. - Jacques Derrida, Elisabeth Roudinesco, De quoi demain..., Paris, Fayard/ Galilée, 2001, p. 90 : « ce qui arrive ou qui fond sur moi, ce à quoi je suis exposé, audelà de toute maîtrise. Hétéronomie, donc, l'autre est ma loi ».

4. - P. Handke, Kali, op. cit., p. 117.

5. - Jacques Lacan, Le Séminaire, II. Le moi dans la théorie de Freud et dans la technique de la psychanalyse, Paris, Seuil, p. 186. 
L'image provient d'un étrange commerce avec l'obscur : renvoyant au travail de la perte de ce qui n'est pas ou plus, l'image ouvre directement accès à une absence et à l'invisible qui n'est pas seulement ce que l'on ne voit pas, mais aussi ce qui ne peut pas être vu, ce qui n'est pas ou plus. À l'image de cette neige sous laquelle n'existe qu'un «entremêlement sans règles », « un éparpillement sans bornes » et qui, «ne [donnant] même pas une impression de neige », ne laisse que des «traînées de nuit, qui traversent maintenant la lumière du jour $»^{6}$, l'image « enveloppe un certain néant $\gg^{7}$ pour reprendre les mots de Sartre, elle trouve dans cette absence son foyer originaire ainsi que le moteur dialectique et psychique de sa dynamique. Loin d'une présence qui illumine et comble, l'image de la catastrophe donne avant tout forme au vide et à l'absence.

\section{L'altérité énigmatique}

Cette terreur qui déclenche le récit transforme le chant de la cantatrice en un cri, expression d'une terreur primaire qu'il faut dépasser pour accéder à la connaissance de soi et de la vérité de ce qui a eu lieu. En effet, les images recèlent un mystère et un secret qui fondent une altérité au sein du moi. Qu'il s'agisse de l'angoisse ou du battement dialectique entre le cône de lumière, l'illumination provoquée par la réminiscence, et son commerce avec l'ombre, la mémoire renferme une altérité énigmatique. L'image renvoie ainsi à un certain « régime du sujet»:

L’image est un régime du sujet [...]: [la subjectivité] y affirme moins sa maîtrise propre qu'elle ne laisse apparaître la façon dont l'altérité la travaille. La subjectivité, quand elle se formule en images, vient s'articuler à l'altérité. Plutôt que l'expression directe du «moi » dont elle signale les leurres, les fêlures ou les tensions internes mieux que la précaire cohérence, l'image serait, en ses récalcitrances mêmes, la traduction du divorce, de l'écart entre $«$ je » et $«$ moi ${ }^{8}$.

Le «son final », arraché à la chanteuse par l'angoisse, est paradoxalement «l'amorce de cette histoire » qui rejoue alors le drame de Shéhérazade, pour qui la parole permet de déjouer et de repousser sans cesse la fatalité qui pèse sur elle et qui la menace de mort.

Tout l'enjeu des deux premières pages de ce texte consistera donc à faire voir cette mémoire qui, trop lourde de l'histoire d'une vie et d'une communauté, devra s'abandonner à un conte qu'il faut dès lors considérer comme le récit d'une mémoire perdue. Dans ce conte, comme dans les

6. - P. Handke, Kali, op. cit., p. 28-29.

7. - Jean-Paul Sartre, L'Imaginaire, Paris, Gallimard, 1986, p. 34.

8. - Jean-Michel Maulpoix, "La Mémoire-cache », in : Murielle Gagnebin, Christine Savinel (dir.), L'Image récalcitrante, Paris, Presses Sorbonne Nouvelle, 2001, p. 173. 
autres, il en va de la survie de l'individu et du groupe, et c'est en passant du singulier à l'universel, à l'archétype et aux structures symboliques de la psyché humaine que s'opère ce processus. Raconter revient alors à rendre visible, à faire venir au jour, à la présence, en le représentant, en le racontant avec les mots du discours, en le traduisant, ce lieu d'origine qui ne peut, par structure, que rester invisible. Apparaît alors peu à peu un décor sur lequel se découpe non pas tant un être qu'une silhouette, un spectre, une ombre, bref une présence absentée, pourrait-on dire : «Et de nouveau je la vois debout, dehors dans une rue, seule. [...] Il est tard dans la nuit, et de nouveau une quasi-clarté de scène sinon de jour $» 9$. Cependant, pour faire face à cette « inquiétante étrangeté » que Freud a théorisée et mise en évidence dans le conte, Peter Handke met en place un dispositif scopique destiné à conjurer la fascination exercée sur le regard. Une fois ce processus amorcé, « la survie devient une question de sur-vision » comme l'explique Derrida dans Parages ${ }^{10}$ et l'exigence de vérité devient rapidement apprentissage, acquisition d'un regard qui triomphe de l'aveuglement et nous fait accéder à l'arrière-scène.

Partant souvent en quête d'un objet perdu afin de réparer un méfait, selon l'analyse proppienne, le conte thématise les relations intergénérationnelles et interroge les relations de filiation souvent défaillantes et à reconstruire. Qu'il s'agisse de la femme en quête de l'enfant perdu, ou de la communauté humaine en proie aux tourments de la guerre, c'est bien la question de la continuité et de la transmission que l'œuvre interroge. La peur renvoie à un fond psychique primaire qui s'élève à l'universel par le truchement de l'anonymat, de l'indifférenciation des personnages. Le véritable événement du conte serait alors la mort apparente de ce double doué d'un nom, d'une histoire, d'un devenir et dont le deuil pourrait justement ré-engendrer la vérité d'un soi sous la forme d'une femme sans passé et sans identité hors du conte. L'auteur fait passer le récit de l'histoire particulière et individuelle - une histoire innommable, irreprésentable, irracontable - au rang de récit universel dans lequel le personnage, la chanteuse en l'occurrence, se défait de ses attributs et caractéristiques personnels pour devenir $«$ celle-ci ou cette autre ${ }^{11}$.

\section{Une écriture de la reprise}

Raconter revient donc, comme pour Shéhérazade, à repousser indéfiniment la mort, à la conjurer par la puissance de la répétition, et fonde, à chaque recommencement, l'unicité d'un «il y a », d'une histoire et d'une singularité. Ce que Peter Handke entreprend, à travers le destin de

9. - P. Handke, Kali, op. cit., p. 11.

10. - Jacques Derrida, Parages, Paris, Galilée, 2003, p. 127.

11. - P. Handke, Kali, op. cit., p. 10. 
cette femme anonyme, c'est de livrer un récit exemplaire à la dimension universelle, afin de refonder, par la reconstruction d'une mémoire individuelle perdue, une mémoire collective qui n'est cependant possible que dans l'avènement toujours renouvelé, répété mais unique d'une voix, d'un «il était une fois ». L'un des enjeux fondamentaux de ce roman correspond donc à la capacité du roman à fabriquer du continu à partir du principe de répétition. Nous abordons là l'un des points essentiels de l'esthétique handkéenne qui renvoie aux structures mythiques mises en œuvre par le conte. Ainsi l'auteur explique-t-il que «le mythe est constitué de répétitions : des événements comparables avec différentes personnes à différents endroits et à différentes époques »12. Cela s'explique par le fait que cet art du récit fondé sur la répétition est une réplique à la mutation des formes de l'expérience du temps amorcée au déclin des Lumières. En réponse au régime moderne d'historicité que Walter Benjamin et Hannah Arendt évoquaient dans les termes d'une crise de l'expérience et d'une rupture de la tradition, les poétiques de la répétition élaborent une singulière politique du deuil selon laquelle le passé qui revient ne réconcilie pas le présent avec l'autrefois, mais fait différer le présent d'avec lui-même.

Cette résistance mélancolique, qui multiplie fantômes, spectres et revenants, permet alors de réinscrire dans le texte l'un des enjeux essentiels du conte et de la quête, à savoir l'énigme. En effet, la répétition, comme nous l'explique Deleuze, produit un télescopage de deux présents : "L'écho des deux présents forme seulement une question persistante, qui se développe dans la représentation comme un champ de problème, avec l'impératif rigoureux de chercher, de répondre, de résoudre ${ }^{13}$. Répondre à l'énigme posée par la répétition et le télescopage temporel revient à définir le type de savoir et de connaissance que délivre le conte non plus en termes kantiens ou cartésiens. Il suffit pour s'en convaincre d'observer attentivement la relation entre l'homme et la femme. Le jeu des questions auquel se livrent les deux personnages finit en effet par s'engager sur le double terrain de la mort et de l'amour que la chanteuse conclut par une longue tirade issue du Parzival de Eschenbach qu'elle commente en ces termes : «Maintenant je te dis : oui, si nous deux, nos deux corps, nous aimons, il nous faut mourir, vous avec moi, et moi avec vous ${ }^{14}$. L'union charnelle advient par le Verbe, dans la parole de la chanteuse et doit être comprise comme un

12. - Peter Handke, Phantasien der Wiederholung, Frankfurt a.M., Suhrkamp, 1983, p. 83 : « Der Mythos besteht aus Wiederholungen: vergleichbare Geschehnisse mit verschiedenen Personen an verschiedenen Orten zu verschiedenen Zeiten ».

13. - Gilles Deleuze, Différence et répétition, Paris, Presses Universitaires de France, 1968, p. 115.

14. - P. Handke, Kali, op. cit., p. 75-76. 
état d'abandon, de complète ouverture à l'autre que soi qui élève l'individu à la véritable connaissance, mais une connaissance érotique, dans la mesure où « la réponse vient toujours d'ailleurs : toute réminiscence est érotique, qu'il s'agisse d'une ville ou d'une femme. C'est toujours Érôs, le noumène, qui nous fait pénétrer dans ce passé pur en soi, dans cette répétition virginale, Mnémosyne. Il est le compagnon, le fiancé de Mnémosyne $»^{15}$.

\section{En route vers l'arrière-pays}

Ce passé pur, immémorial, inexistant et synonyme d'Oubli, c'est ce que le narrateur appelle "l'arrière-scène », et qui renvoie en réalité à ce point d'origine de l'individu et du récit, à savoir l'arrière-pays de l'Enfance qui ne doit pas être considérée ici comme une donnée dont on pourrait isoler le lieu chronologique, ni comme un âge ou un état psychosomatique de l'individu. L'Enfance n'est pas un lieu, ni un temps facile à déterminer, elle échappe au contraire à toute catégorie spatiotemporelle et se caractérise par sa mouvance, son indétermination même. Ainsi la chanteuse peine-t-elle à expliquer au guitariste l'endroit où elle se rend:

Dans la région de mon enfance. Ou non dans la région voisine. Dans la région tout à côté, derrière la colline de mon enfance. Dans la région derrière ma région. Partout ailleurs, j'ai l'impression de m'y être déjà trouvée, en Alaska, en Guadeloupe, à Madagascar, partout ici aussi en Europe, même en Suisse et en Autriche. Mais le pays au-delà de la forêt d'enfance je l'ai autrefois tout au plus traversé ${ }^{16}$.

Face au monde de l'indétermination et de l'impossibilité à assigner un sens au réel, le regard handkéen mobilise une autre forme de savoir, un savoir paradoxal reposant sur l'idiotes, c'est-à-dire, si l'on se réfère à l'étymologie de ce mot comme le fait Clément Rosset ${ }^{17}$, à la simplicité et à l'unicité d'un monde saisi dans ses singularités. L'écriture consistant pour Peter Handke dans la capacité à « retrouver, par le déchiffrement, l'enfance ${ }^{18}$, elle rejoint le « besoin en tant qu'auteur d'inventer et de trouver des mythes $»^{19}$, une origine qui se situe en-deçà ou au-delà du sujet, à un niveau pré-individuel puisque renvoyant à l'expérience de l'enfant qui ne doit pas être considéré comme l'enfant Peter Handke,

15. - G. Deleuze, Différence et répétition, op. cit., p. 115.

16. - P. Handke, Kali, op. cit., p. 25.

17. - Clément Rosset, L'Objet singulier, Paris, Éditions de Minuit, 1979.

18. - Peter Handke, Am Felsfenster morgens, Salzburg, Residenz, 1998, p. 267 : «Entzifferndes Wiederfinden der Kindheit ».

19. - Peter Handke, Das Gewicht der Welt, Salzburg, Residenz, 1997, p. 160 : « das Bedürfnis, als Schriftsteller Mythen zu erfinden, zu finden». 
mais comme un rapport au monde caractérisé par un état de naïveté créatrice. Nous retrouvons alors l'importance de la naïveté qui caractérise tant de personnages de conte. En effet, le seul mode d'accès et de compréhension du réel est donc, paradoxalement, l'ignorance, le nonsavoir dont il faut faire l'éloge. Or cela n'est possible que par le geste profondément didactique et naïf que constitue le regard de l'enfant et qui amorce le retour à un état natif de la perception et du rapport au monde. La distanciation et la défamiliarisation opérées par le regard de l'enfant rompent avec l'idée d'une fixité et d'une identité des choses pour faire place à l'altération et aux métamorphoses du réel dont le principal effet est de produire de l'étrangeté.

Il apparaît alors que ce procédé permet de donner une tout autre dimension au deuil. En effet, celui-ci ne doit plus être considéré comme un travail destiné à combler le vide laissé par la perte de l'être aimé, mais, à l'instar de Jean Allouch, comme un " gracieux sacrifice $»^{20}$. Ainsi, apparenter le deuil au sacrifice, plutôt qu'à un travail psychologique, revient à l'appréhender comme le spectacle d'un anéantissement qui rend le sujet à l'intimité dérobée du monde. Ce roman, dans la fidélité à l'expérience de l'effroi, de la terreur et du deuil dont il naît, se donne donc comme le lieu d'un interminable sacrifice, d'une mort apparente de soi qui ne vient pas rétablir l'ordre défait du monde, mais vient réveiller un désastre devenant à son tour expérience de beauté et de vérité. Si le conte est répétition, reprise, il est aussi revenance dans ce roman. La chanteuse, une fois sacrifiés sa vie et son passé, prend l'aspect d'un spectre revenant sur les lieux de son origine. Kali n'a pas seulement une existence géographique (vérifiable) ou symbolique (l'arrière-pays de l'Enfance), cette ville est aussi le lieu d'un désir et le récit relate le mouvement nocturne qui reconduit vers ce lieu tout en faisant se dissiper son ombre dans l'espace sans fond du monde. Survivante, la protagoniste, en effectuant ce voyage assimilable à une expérience sacrificielle, s'en revient vers la vie pour se perdre dans l'intimité extatique du monde, dont Bataille dit qu'elle est jouissance et absence d'individualité.

\section{La mouvance du monde}

À travers le parcours régressif de la protagoniste sur les terres de son enfance, l'auteur met en place un espace où les formes peuvent s'échanger et se métamorphoser. Nous touchons à la puissance proprement imaginative du conte dont l'entreprise de déréalisation donne lieu à des mécanismes métamorphiques mis en valeur par le narrateur luimême, reposant sur le principe analogique, et à des actions imaginaires

20. - Jean Allouch, Érotique du deuil au temps de la mort sèche, Paris, EPEL, 2011, p. 9-10. 
où s'estompe toute dualité catégorielle. Les personnages répondent à une logique de poupée gigogne, brouillant des identifications parfois bivalentes et réciproques. Tout peut alors s'inverser en figurant son envers ou son endroit. Le conte dans ce texte se caractérise essentiellement par sa dimension métaphorisante au sens où le conte, comme l'explique Fédida:

par le fonctionnement de sa métaphore, reconstruit chaque fois l'événement que l'angoisse rend innommable. Les dangers intérieurs qui surviennent « entre chien et loup », au moment de l'endormissement ou dans le silence de la béance, appellent la métaphore comme le seul espace $[\ldots]$ où les formes peuvent s'échanger et se métamorphoser, où les actions imaginaires peuvent s'inverser en figurant leur envers et leur endroit, où enfin les personnages sont un seul et même personnage qui permet des indications toujours bivalentes et réciproques ${ }^{21}$.

Nous retrouvons, contenus dans cette citation, les principaux éléments de réflexion destinés à éclairer le principe analogique au fondement de la reconstruction/création d'un monde oublié et rendu plausible.

La métamorphose est l'un des procédés les plus fréquents et les plus souvent évoqués dans l'œuvre de Peter Handke. L'espace intervallaire de l'endormissement propre au conte, l'espace de la béance ne signifie donc pas seulement l'absence ou la privation de lieu. Au contraire, la neutralité est bien plutôt synonyme de positivité et de productivité : l'évidement suscite le remplissage, voire le trop-plein, créant alors un espace que l'on peut décrire comme l'espace à la fois de la potentialité et de l'absence. S'inspirant du romantisme allemand, l'auteur suit une tendance très novalisienne de « potentialisation qualitative » 22 qui met en place un jeu de substitutions entre les personnages et le narrateur. Les procédés de transformation et de substitution s'articulent étroitement l'un à l'autre. Par son pouvoir métamorphique, le sujet d'écriture se mue en cellule germinale à partir de laquelle se déploie le jeu des substitutions, révélant une logique de prolongement/dépassement au cœur des relations entre le sujet d'écriture, les personnages et les objets du monde, dévoilant alors la nature mythique d'un sujet transfiguré dans ses simulacres. Ces expériences métamorphiques surviennent et s'accumulent à la fin du roman, s'imposant abruptement au narrateur qui ne peut que constater : " une métamorphose », " et encore une métamorphose », pour ensuite laisser place à la description de l'expérience existentielle et visuelle qui se produit :

21. - Pierre Fédida, Corps du vide et espace de la séance, Paris, MJW Fédition, 2012, p. 148.

22. - Novalis, Le Monde doit être romantisé, trad. Olivier Scheffer, Paris, Allia, 2002, p. 46. 
On ne voyait pas encore de neige dans la région vers laquelle elle était partie. Ce fut seulement dans une flaque à ses pieds que planèrent quelques flocons, sur quoi, du fond de la flaque, une bulle s'éleva. Et puis un essaim de flocons atterrit sur une langue de sable, fondit sur-le-champ et forma sur le sable clair des cercles qui allaient en s'agrandissant. Elle tendit une plume d'oiseau, que traversait la lumière, vers le ciel, comme pour prendre la mesure, puis souffla dans son harmonica : un appel, encore et encore, qui ne pouvait pas faire peur, même au plus craintif des oiseaux ${ }^{23}$.

L'enjeu essentiel de ces quelques lignes, et de toute métamorphose, est, selon la formule de l'auteur, de « rendre connu l'inconnu; arpenter l'espace de l'inconnu et l'agrandir ${ }^{24}$. Il s'agit de prendre la mesure du monde et de s'affranchir de la pesanteur des choses. Aux prises avec l'indéterminé, le sujet d'écriture se déploie, se déplace et se transmue dans le texte à travers la «multiplicité des résonances » 25 qui se déploient en orbes pour s'élever peu à peu aux dimensions de l'univers et atteindre enfin un sentiment d'harmonie et de paix avec le monde. Mouvant et protéiforme, le sujet d'écriture caméléon s'approprie certaines caractéristiques de la pensée mythique dont la singularité tient justement à sa grande malléabilité ainsi qu'à sa propension à se répéter dans la différence. Il se révèle être un foyer d'échos privilégiant l'équivocité, à l'image d'un sens introuvable et cependant central qui, par son « épaisseur sémantique », « déborde de toutes parts la linéarité du signifiant » et qui transforme le sujet d'écriture en un « palais des miroirs dans lequel chaque mot renvoie en tous sens à des significations cumulatives ${ }^{26}$. À travers l'image se jouent des procédés de déplacements de condensation, voire de dramatisation qui produisent un «spectre d'identité » au long duquel, dans les franges, se répartit le « je » qui n'est pas dans le «Moi ». Dans ces textes, la voix qui résonne d'écho en écho fonde une parole qui, telle la Chose héraclitéenne et présocratique, nourrit la dialectique du déterminé et de l'indéterminé. En plus du jeu de substitutions qu'elles mettent en place, les métamorphoses du sujet d'écriture bouleversent les codes de l'identité et de l'écriture de soi en niant tout principe de vérité, de causalité et nous obligent à ne plus penser en termes de contradictions ni d'oppositions, mais de juxtaposition ou de succession.

23. - P. Handke, Kali, op. cit., p. 114-115.

24. - Peter Handke, Die Wiederholung, Frankfurt a.M., Suhrkamp, 1986, p. 262 : «Bekanntes unbekannt machen; den Bereich des Unbekannten abschreiten und vergrößern ».

25. - Roger Caillois, Le Mythe et l'homme, Paris, Gallimard, 1987, p. 30.

26. - La comparaison est de J. Soustelle, cité par Gilbert Durand, Les Structures anthropologiques de l'Imaginaire, Paris, Dunod, 1981, p. 413. 


\section{L'épreuve de l'inouï}

La régression ne saurait donc plus se définir simplement à partir des stades de développement auxquels quelque chose ferait retour, elle doit aussi être conçue « comme l'imagination que se donne le vivant » et qui « fait appel à des déplacements de lieux et des dyschronies » 27 . Une telle conception nous invite à entrer en contact avec ces contenus régressifs et à construire les formes de la régression, mouvement qui est appelé « l'informe », d'après Bataille. Ce qui s'exprime dans le texte, c'est un désir de figurer qui se trouve confronté à un matériau qui ne s'y prête pas spontanément (les pensées latentes du rêve, la surface plane du tableau). Dans les deux cas, cette difficulté est résorbée par une défiguration appropriée: il s'agit de figurer malgré tout, de forcer à la figuration, de creuser la représentation, même au prix d'une certaine étrangeté. Dans les deux cas, la figure est un détour pour faire accéder à ce quelque chose qui est présent virtuellement mais qui n'est pas représenté. Ce quelque chose, il est impossible de le saisir durablement. Il se présente comme oubli, non-savoir, dans une singularité qui ne se dénude pas.

Reprenant le chronotope de la rencontre que Bakhtine analyse justement dans le Parzival de Eschenbach, Peter Handke élabore le texte comme un seuil traversé par les trajectoires de ses personnages, programme annoncé dès le début par la chanteuse qui n'avait fait que traverser la « région d'avant la forêt ». Les personnages, comme dans les autres textes de l'écrivain, apparaissent, se rencontrent, se croisent, creusent des chemins, forment des couples, ou des groupes. Ils sont décrits minutieusement selon le type de mouvement, les micro-actions et les accessoires, le rythme, l'ampleur du geste et l'humeur de son exécution, jusqu'à arriver à des mouvements d'ensemble, comme dans la scène suivante :

Comme nombreuses étaient les choses étranges avec elle : elle rencontra un jeune couple, en pleine lande, main dans la main, simplement, comme cela, sans sac à dos, sans chien, sous le ciel, et on se salua, il resta un instant de joie commune, et on s'éloigna sous le ciel. De même ensuite un couple âgé, et ce couple n'avait rien d'autre en tête que d'aller ensemble. Un coureur, qui en courant se balançait, sans écouteurs, sans musique. Un randonneur à vélo qui, débouchant d'un tournant de la forêt, sans maillot, sans casque, sans éclaboussures dans le dos, ralentit devant elle, traçant une boucle attentive autour d'elle, et salua $^{28}$.

27. - Pierre Fédida, Par où commence le corps ? Retour sur la régression, Paris, Presses Universitaires de France, 2000, p. 12.

28. - P. Handke, Kali, op. cit., p. 113. 
Tout cet entrelacs de mouvements et de trajectoires permet donc à l'auteur de réintroduire une forme de contingence, de hasard qui se rapprocherait de la tuchê grecque dans la mesure où elle signalerait l'effraction de la réalité, la rencontre du réel, autrement dit ce qui, sous la forme de « voix, sonorités, ou timbres [venus] d'un autre temps $»^{29}$, littéralement nous atteint, nous touche, choit, nous échoit par-delà les époques et qui nous destine dans l'instant même d'une rencontre avec l’imprévu, le non-visible par excellence.

C'est pour l'auteur une manière de retrouver une nouvelle forme d'aventure, car c'est dans le hasard de la rencontre, dans l'expérience de la tuchê qu'elle se situe. L'apprentissage comme la quête privilégient ici la structure narrative d'un texte dont il faut souligner le fonctionnement paradoxal particulièrement visible à la fin du texte. En effet, la narrativité laisse place à une accumulation de scènes reliées entre elles par le principe de succession et que le narrateur introduit très sobrement par des formules à peine variées telles que « S'ensuivent de petites scènes », "S'ensuivit une scène dans laquelle », "Ensuite une scène » ou bien encore «Puis encore une scène $»^{30}$. Il apparaît progressivement au fil de l'œuvre que ce n'est pas la narration qui constitue la structure de base du roman. En effet, il ne s'agit pas tant de mener de bout en bout une histoire enchaînant des événements selon le principe de causalité qui transforme les personnages dans le temps, que de mettre en place une logique plus séquentielle propre à l'économie de l'aventure : c'est dans la rencontre que s'effectuerait la mise à l'épreuve. L'importance accordée à la manifestation du réel instaure, dans la contingence du monde et l'aléa des rencontres inouïes, une cohérence interne : le merveilleux et le surnaturel se juxtaposent sans conflit à la réalité et font désormais partie de l'ordre du monde.

\section{Reconstruire l'événement}

Ce qui se joue dans cette logique de la rencontre et de l'épreuve, c'est la capacité du roman à reconstruire l'événement. Or cette fonction du récit est directement liée à la voix du conteur, dans la mesure où le conte crée un espace vocal dans lequel la parole, par le fonctionnement de sa métaphore, reconstruit chaque fois l'événement que l'angoisse rend innommable. Pour ce faire, Peter Handke recourt à des procédés déjà évoqués tels que la répétition, et qui s'avèrent être de véritables

machinations d'écriture [...] qui n'ont peut-être d'autre effet que de laisser entendre dans de surprenantes figures une voix brouillée, excommuniée, de personne ; plus ancienne que les souvenirs parce qu'elle

29. - Ibid., p. 114.

30. - Ibid., p. 103-106. 
est l'écho de cette écriture. D’où ces essais de mémoires nés de cette écoute, qui s'écoutent «ainsi » parfois $^{31}$.

Cette voix, ce cri n'est en réalité qu'un écho, c'est l'écho du réel, de cet impossible qui nous adresse un appel qu'il faut considérer comme le lieu d'un défaut, d'une déchirure. Face à un réel qui s'avère irreprésentable par le biais mimétique, qu'il est tout simplement impossible de refléter, de représenter. Ce manque, cette déchirure, il nous faut l'approcher par le détour de la fiction et du conte, non seulement en déréalisant et en métamorphosant le réel, mais aussi en reproduisant en retour l'écho d'une parole (que ce monde perdu, que cette expérience perdue même, nous adresse) qui rend à nouveau audible ce monde.

Une telle entreprise n'est possible que parce que la dynamique de l'épos propre au conte possède la capacité de faire surgir l'image du souvenir comme "action arrivante", ce qui libère le récit «dont la diachronie n'est pas chronothétique (séparatrice d'époques), mais aspectuelle $»^{32}$. Il devient alors possible par ce biais de peupler les « coins morts » de cette région, comme ce bar où il n'y a « encore personne à voir » et pas de musique jusqu'à ce qu'il commence à se remplir et

après une brève absence d'événements entrèrent les uns après les autres plusieurs silhouettes de joggers, avec des visages égratignés, comme aveuglés par tant de lumière après un assez long moment dans les ténèbres de la forêt. Ils en arrivaient avec tout ce qui aurait pu manquer pour un festin ; l'apportaient presque en excès, mais aussi comme un par un, comme une trouvaille, comme quelque chose de minuscule ${ }^{33}$.

Il ne s'agit pas ici pour le narrateur de déterminer une cause, de faire jouer des rapports de causalité, de représentation et de signification «impliquant des intentionnalités discursivement objectivantes », mais une signifiance, comme si elle «supposait des formes en procès d'énonciation au lieu de signes en opération de signification $»^{34}$. En déconstruisant la logique syntaxique horizontale et causale de la langue de la communication et de la signification, « le mot retrouve sa force de nom [...], et il retrouve cette forme de nomination parce que la chose l'imagine $»^{35}$. Le texte se trouve alors prêt à recevoir l'incidence pure de l'événement grâce à l'avènement de la parole nominale.

31. - Louis Marin, La Voix excommuniée, Paris, Galilée, 1981, p. 24.

32. - Henri Maldiney, Aîtres de la langue et demeures de la pensée, Lausanne, L'Âge d'Homme, 1975, p. 83.

33. - P. Handke, Kali, op. cit., p. 101.

34. - Pierre Fédida, « Passé anachronique et présent réminiscent. Epos et puissance du langage », L'Écrit du temps, n 10, 1985, p. 26-27.

35. - Ibid., p. 34. 
Du cri primaire proféré au début de l'œuvre à cette voix que le narrateur « [entend] tout de même encore [...] chanter : "Ah, quand un enfant se met à raconter : marche de gens sous les arbres en fleurs"... $»^{36}$, le roman retrace, à travers les épreuves endurées par la chanteuse, le cheminement vers et dans la parole, une parole originaire et archaïque qui correspondrait à celle de l'enfant, infra- ou supra-individuelle, qu'il faut exhumer des replis de l'être. La capacité recouvrée et renouvelée de raconter des histoires ne signifie cependant pas la réappropriation d'une biographie ni la reconquête d'une expérience authentique, car, comme Giorgio Agamben l'explique,

[à] l'expropriation de l'expérience, la poésie répond en faisant de cette expropriation une raison de survivre, transformant en norme de vie ce qui ne peut être expérimenté. Dans cette perspective, la recherche du « nouveau » n'apparaît plus comme la recherche d'un nouvel objet d'expérience ; elle implique, au contraire, une éclipse et un suspens de l'expérience. Est nouvelle la chose dont on ne peut faire l'expérience, parce qu'elle gît « au fond de l'inconnu » : la chose en soi kantienne, l'inexpérimentable comme tel ${ }^{37}$.

Face à l'appel de l'impossible, il n'est qu'une réponse, qu'une attitude envisageable : la survie. Dans ce roman, le conte n'apparaît pas tant comme un lieu transitionnel de médiation ou d'endormissement qui atténuerait une peine : au contraire, il invite à répondre à l'appel de l'impossible proféré par le réel et la mémoire par une esthétique de la survie, une esthétique qui permet de « vivre là où le monde est bouleversant » 38 afin de transformer l'expérience traumatique en un contre-monde fictif ouvrant sur l'avenir et une promesse de bonheur.

\section{Conclusion}

Le rapprochement entre cette œuvre de la littérature moderne marquée par la perte et le genre du conte provient certes de l'intérêt de Handke pour ce type de textes, mais il est aussi caractéristique d'une modernité qui porte en elle, presque comme sa loi, la fêlure d'une perte. Cette perte serait ce qui oriente, sinon accomplit, l'expérience des écrivains modernes, qui cherchent leur parole précisément dans le questionnement de la parole. Un dédoublement se trouve au cœur d'une telle expérience poétique, dans l'usage de la parole se constate l'impossibilité d'une parole pleine, consacrant par ce biais la limite du langage et la

36. - P. Handke, Kali, op. cit., p. 118.

37. - Giorgio Agamben, Enfance et histoire : destruction de l'expérience et origine de l'histoire, trad. Yves Hersant, Paris, Payot-Rivages, 2000, p. 53-54.

38. - P. Handke, Kali, op. cit., p. 117. 
situation énigmatique de toute écriture. Partagée entre la vocation de dire et la volonté d'effacement, entre la hantise d'un solipsisme radical et le désir de partage, elle habite un espace où la littérature est sans cesse portée à se questionner sur ce qui l'origine, sur ses latences et ses possibles. Une éthique de l'écriture au plus haut degré d'exigence et de lucidité est au travail dans cette œuvre qui se veut aussi véritable exploration de soi. En ce sens, le conte est aussi ouverture à l'imaginaire et à de nouvelles possibilités de vision. L'espace « aéré » et vide devient un espace intervallaire, marqué par l'indétermination et dont la temporalité mythique scandée par les passages porte en elle des poussées imaginatives qui élèvent le texte aux deux dimensions du non-temps : l'infini de la permanence et le scintillement de l'instant capable de capter la mémoire et la projection dans la présence. Dans cette optique, le conte mobilise nombre de ses procédés caractéristiques pour participer à l'exploration de l'être par les pouvoirs de l'imaginaire et remplacer par ce biais une esthétique du deuil par une esthétique du mystère afin de réaliser dans la beauté de la parole qui énonce la «plénitude de l'amour, plénitude du deuil, plénitude du temps ${ }^{39}$. 
